\title{
Different phases of aging in mouse old skeletal muscle
}

Research Paper

\author{
Yong-Kook Kang ${ }^{1,2}$, Byungkuk Min ${ }^{1}$, Jaemin Eom ${ }^{1,2}$, Jung Sun Park ${ }^{1}$ \\ ${ }^{1}$ Development and Differentiation Research Center, Korea Research Institute of Bioscience Biotechnology (KRIBB), \\ Yuseong-Gu, Daejeon 34141, South Korea \\ ${ }^{2}$ Department of Functional Genomics, Korea University of Science and Technology (UST), Yuseong-Gu, Daejeon \\ 34113, South Korea
}

Correspondence to: Yong-Kook Kang; email: ykkang@kribb.re.kr

Keywords: skeletal muscle, early and late phase aging, transcriptome, young-old, old-old

Received: June 23, $2021 \quad$ Accepted: December 3, $2021 \quad$ Published: January 11, 2022

Copyright: (C) 2022 Kang et al. This is an open access article distributed under the terms of the Creative Commons Attribution License (CC BY 3.0), which permits unrestricted use, distribution, and reproduction in any medium, provided the original author and source are credited.

\section{ABSTRACT}

With a graying population and increasing longevity, it is essential to identify life transition in later years and discern heterogeneity among older people. Subclassifying the elderly population to inspect the subdivisions for pathophysiological differences is particularly important for the investigation of age-related illnesses. For this purpose, using 24- and 28-month-old mice to represent the "young-old" and "old-old", respectively, we compared their skeletal muscle transcriptomes and found each in a distinct stage: early/gradual (E-aging) and late/accelerated aging phase (L-aging). Principal component analysis showed that the old-old transcriptomes were largely disengaged from the forward transcriptomic trajectory generated in the younger-aged group, indicating a substantial change in gene expression profiles during L-aging. By calculating the transcriptomic distance, it was found that the 28-month group was closer to the two-month group than to the 24-month group. The divergence rate per month for the transcriptomes was the highest in L-aging, twice as fast as the rate in E-aging. Indeed, many of the L-aging genes were significantly altered in transcription, although the changes did not seem random but rather coordinated in a variety of functional gene sets. Of 2,707 genes transcriptionally altered during E-aging, twothirds were also significantly changed during L-aging, to either downturning or upturning way. The downturn genes were related to mitochondrial function and translational gene sets, while the upturn genes were linked to inflammation-associated gene sets. Our results provide a transcriptomic muscle signature that distinguishes old-old mice from young-old mice. This can help to methodically examine muscle disorders in the elderly.

\section{INTRODUCTION}

As demographic aging continues, the population structure is shifting to an uncharted phase in which the "super elderly" (80 years and older) outnumber the "elderly" (60 years and older). Older adults are at risk of developing age-associated diseases, so this transition heavily weighs on government finances. Thus, maintaining adult health while aging is important, both for individual quality of life as well as costs to healthcare systems [1].

With a graying population and increasing longevity, it is important to identify life transitions in later years and recognize heterogeneity among older people [2]. The term "late life" is broadly defined by encompassing a heterogeneous group of adults of 65 years and older; hence, it is further classified into "young-old" and "old-old" groups [3, 4] in the hope of identifying the group with a distinct vulnerability to certain chronic diseases and mental illnesses. Supportively, several studies have discerned a comprehensive difference across physical, cognitive, and psychosocial domains between the young-old (aged $60-74$ years) and old-old (aged 75 years and older) groups $[2,5,6]$. A similar distinction may exist for physiological and pathological domains, such as chronic illnesses (cardiovascular disease, cancer, 
chronic respiratory diseases, and diabetes, among others) and the deterioration of skeletal muscle and cognitive function [7]. In reality, these age-related illnesses vary markedly and can, with age, take the shape of a comorbidity, which is the co-existence of two or more diseases [8]. For instance, only $30 \%$ of adults aged $45-64$ years have at least two chronic conditions, whereas $65 \%$ of those aged $65-84$ years and approximately $80 \%$ of those aged 85 years and older have the same conditions [9]. Therefore, to investigate these age-associated diseases, it may be beneficial to divide the elderly into groups and inspect the resultant subgroups separately for pathophysiological differences, and other deteriorations or weaknesses.

In the case of mice, those ranging from 18 to 24 months-of-age, which is comparable to humans of 56 - 69 years-of-age, fulfil the requirements of "youngold" age, whereas mice aged 26 months and older can be considered as "old-old" [10]. It is notable that $22-$ 24 months of age is when morphological changes consistent with human sarcopenia [11] commence in mice and rats $[12,13]$. This is the period skeletal muscle mass and grip strength decline progressively with age, exhibiting prominent changes at 24-28 months of age, while whole-body mass and lean mass were relatively stable or only marginally declined [13]. Another significant distinction between the young-old and old-old groups is survivorship; 24 - and 28 -month-old mice exhibit $85 \%$ and $50 \%$ survival rates, respectively $[14,15]$, or less depending on the strain and sex (Strain survival information, https://www.nia.nih.gov). Based on this rapid declines in muscle mass and survivorship with age, we assumed that aging accelerates in "late life" in a manner different from that in the slow aging mode before then. In addition to the increased morbidity and accelerated aging, we recently noticed that skeletal muscle in old-old mice, but not in young-old mice, underwent DNA demethylation particularly over genomic retroelements, and as a consequence, a large number of genomic retroelement copies acquire the competence for transcription [16]. Similarly, the existence of other unexplored molecular and physiological traits that distinguish old-old mice from young-old mice, is also conceivable.

Recently, there were reports of bulk [17] and singlecell RNA-seq analysis [18] on aging hallmarks across the organs and age in mice, but the global scale in these studies has benefit for understanding overall picture of aging but is not greatly helpful to study in detail aging events particularly in skeletal muscle and in "later life" if not thoroughly re-analyzed. We examined the transcriptomes of skeletal muscle sampled from 24- and 28-month-old mice as the young-old and old-old groups, respectively, along with 2-, 10-, and 18-month-old mice representing young and midlife controls. By focusing on the genes that deviated from the normal expression profiles in the late aging phase, our study provided insight into the transcriptomic features of the skeletal muscle of oldold mice compared to that of young-old mice. Furthermore, we investigated whether there are any genes in which the expression shifts are coordinately regulated in accordance with the transition in "late life"; thereby, providing a thorough impact assessment of the late aging phase.

\section{RESULTS}

\section{Comprehensive gene expression changes during late phase of aging}

We obtained RNA sequencing data from the skeletal muscle of 2- $(2 \mathrm{~m} ; \mathrm{n}=5), 24-(24 \mathrm{~m} ; \mathrm{n}=6)$, and 28 month-old mice $(28 \mathrm{~m} ; \mathrm{n}=4)$ to compare their transcriptomes. We regarded 24 and 28 months-of-age as young-old and old-old and provisionally designated the corresponding periods as the early aging (E-aging) and late aging phase (L-aging), respectively (Figure 1A and Introduction for the rationale). Principal component analysis (PCA) result showed that the transcriptomes of young, young-old, and old-old age groups clearly diverged from each other (Figure 1B). In total, 707 differentially expressed genes (DEGs; fold-change $>2$ and $P<1 \times 10^{-5}$; Supplementary File 1) were identified in the comparison of $28 \mathrm{~m}$ and $24 \mathrm{~m}$ samples. This DEG number was considerable for a short period of only four months, and comparable to the 1,394 DEGs detected in the 24m-versus-2m comparison (Figure 1C and Supplementary File 1). This indicated that global gene expression changes occur during L-aging. We confirmed differential expressions of the identified DEGs in the young-old and old-old muscle samples through a quantitative real-time PCR (Supplementary Figure 1).

\section{Some cellular processes were disturbed in a coordinated and L-aging-specific fashion}

We inspected the young-old and old-old muscle transcriptomes in detail, to discover genes whose expression was changed in a coordinated L-agingspecific manner, thus revealing certain cellular events disturbed during L-aging. We performed gene set enrichment analysis (GSEA) on the "HALLMARK," "KEGG PATHWAY," and "GO.BP" collections using the fast pre-ranked GSEA (fGSEA) package, to interpret coordinate changes in the transcriptomes of $28 \mathrm{~m}$ over $24 \mathrm{~m}$ samples (Supplementary File 2). A 
number of gene sets were significantly enriched or depleted in the $28 \mathrm{~m}$ transcriptomes (Figure 2A and Supplementary Figure 2). The significantly enriched genes were associated with cell adhesion, cell signaling pathways, and inflammation-related sets, whereas the significantly depleted ones were linked to oxidative phosphorylation and translation terms. Single-sample GSEA (ssGSEA) using gene set variation analysis (GSVA) was performed, which assesses separate enrichment scores (ESs) for each sample and gene set pairing, to determine the extent of coordinate gene set up- or downregulation within a sample group [19]. GSVA results demonstrated that the $28 \mathrm{~m}$ samples were synchronized in expression levels for a variety of GO.BP gene sets $(\mathrm{n}=384)$, showing a pattern opposite to that of the $24 \mathrm{~m}$ samples $\left(P_{\text {adj }}<1 \times 10^{-5}\right.$ and $\log 2$ fold-enrichment $>0.5$; Figure 2B and Supplementary Figure 3). Notably, genes were overrepresented in the $28 \mathrm{~m}$ samples in the majority of the selected terms $(89.3 \%, 343 / 384$ terms $)$. Our results indicated that the old-old transcriptomes are distinguishable from the young-old ones and that the transcriptomic change in L-aging might not be totally fortuitous but predictable to some degree for certain gene sets. The same gene sets selected by the bulk GSEA (Figure 2A and Supplementary Figure 3) were reproducibly chosen from the GSVA.

Transcriptomic distance among the young, youngold, and old-old in skeletal muscle and blood cells

For evenly spaced chronological transcriptomes, middle-aged samples were required. Therefore, we carried out RNA-sequencing of the skeletal muscle from 10- and 18-month-old mice $(\mathrm{n}=6)$. In a PCA, using the sequencing data of all age groups, we discovered that the $24 \mathrm{~m}$ transcriptomes slightly deviated from the forward trajectory and that the $28 \mathrm{~m}$ transcriptomes swerved completely outward; thus, both these groups disengaged from the transcriptomic path of younger age groups (Figure 3A). We measured the transcriptomic distance between the age groups based on Euclidian distance [20]. The $24 \mathrm{~m}$ group was the furthest cluster from the $2 \mathrm{~m}$ group, whereas the $28 \mathrm{~m}$ group was the nearest (Figure 3B). The $10 \mathrm{~m}$ and $18 \mathrm{~m}$ transcriptomes clustered close to those of the $24 \mathrm{~m}$ on the PCA plot. Interestingly, the results indicated that the old-old transcriptomes clustered closer to the young ones than the young-old ones. The monthly divergence of the transcriptome with age, calculated by dividing the transcriptomic distance between the age groups by the age difference in months, decelerated until 18 months and then accelerated as the tissue entered into the E-aging and L-aging groups (Figure 3C). The divergence rate in the L-aging period was approximately twice as high as the rate in the Eaging period (10.6 versus 4.8).

We examined peripheral blood mononuclear cells (PBMCs) to test the generality of our findings with skeletal muscle. In the PCA results, using RNA-seq data of PBMCs obtained from the same mice as that used for muscle tissue collection, the transcriptomes of different age groups demonstrated a boomerang-shaped shift with age (Figure 3D). As for transcriptomic distance, relative to the $2 \mathrm{~m}$ group in the PBMCs, the $28 \mathrm{~m}$ group was the nearest (Figure $3 \mathrm{E}$ ), and the monthly divergence rate was the highest for L-aging, similar to that in the skeletal muscle (Figure 3F). Collectively, our results indicated that the transcriptomes are markedly altered during L-aging, with the trajectory steeply disengaged from the initial forward path. With regards to the gene
A

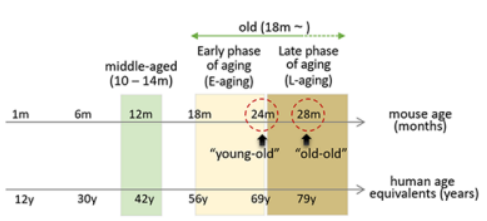

B

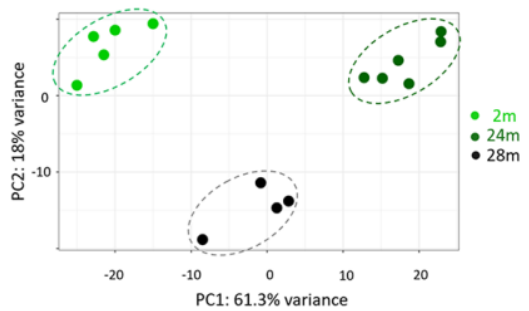

C

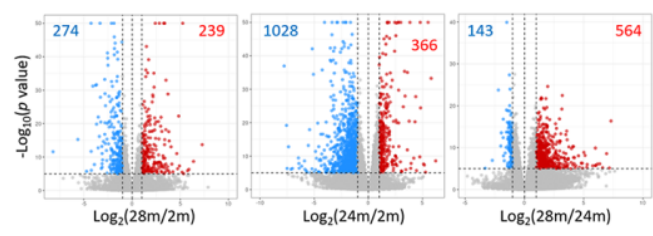

Figure 1. Comparison of skeletal muscle transcriptomes of 2-, 24-, and 28-month-old mice. (A) Representative age ranges for mature life history stages in C57BL/6J mice are shown, together with human age equivalents: 10 to 14 months-of-age as middle age and 18 24 months as early phase of aging (E-aging) [10]. We added the life phase of $25-28$ months or older as late phase of aging (L-aging); note that we substituted the "old" definition in the online article [10] with E-aging. We named 24 and 28 months-of-age as "young-old" and "oldold". Aging phases are shaded in different colors. (B) Principal component analysis (PCA). Transcriptomes of different age groups are marked as dots and lines with different colors. $2 \mathrm{~m}, 24 \mathrm{~m}$, and $28 \mathrm{~m}$ stand for 2-, 24-, and 28-month-old mice, respectively. (C) Volcano plots for comparison of gene expression between $2 \mathrm{~m}$ versus $28 \mathrm{~m}$ (left), $2 \mathrm{~m}$ versus $24 \mathrm{~m}$ (middle), and $24 \mathrm{~m}$ versus $28 \mathrm{~m}$ (right). Differentially expressed genes $\left(\log _{2}\right.$ (fold change $>1.0$ and $p<1 \times 10^{-5}$ ) are colored. 
expression profile, the old-old group clustered closer to the young one than the young-old group in both the skeletal muscle and PBMCs.

\section{Transcriptomic muscle signature discriminates the old-old from the young-old}

To specify the genes that underwent transcriptional alterations during E-aging and L-aging, we classified them based on expression pattern into three types: E-aging, L-aging, or EL-aging genes. Expression levels of the first two types were significantly altered $\left(P_{\text {adj }}<0.001\right)$ in each designated period only but not in the other period $\left(P_{\text {adj }}>\right.$ 0.1; Supplementary File 2). In contrast, EL-aging genes showed significant changes in both the E-aging and Laging periods in a row (Figure 4A). In total, 1,676 ELaging genes were identified that accounted for $9.5 \%$ of all genes. Most of the EL-aging genes (except for seven and 15 genes continuously increasingly and decreasingly expressed, respectively (see [21] for the case of rat limb muscle) showed fluctuations in their expression levels, either an up-and-down (downturn; 785 genes) or a down- and-up (upturn; 869 genes) pattern at 24 and 28 months, starting at two months (Figure 4B and Supplementary File 2). Gene ontology (GO) analysis using the downturn genes, revealed that the terms were primarily related to mitochondrial function and translation involving "mitochondrial ATP coupled electron transport (GO:0 042775; $\left.P_{\text {adj }}=8.7 \times 10^{-48}\right)$," "translation (GO:0006412; $\left.P_{\text {adj }}=2.3 \times 10^{-31}\right)$," and "mitochondrial transport (GO:0006839; $P_{\text {adj }}=7.8 \times 10^{-22}$ )," among others (Figure 4C and Supplementary File 2). GO analysis using the upturn EL-aging genes yielded terms that are related to immune reaction and cell signaling, including "neutrophil-mediated immunity (GO:0002446; $P_{\mathrm{adj}}=3.9$ $\times 10^{-21}$ )," "extracellular matrix organization (GO:0030198; $\left.P_{\text {adj }}=1.1 \times 10^{-11}\right)$," and "platelet degranulation (GO:0002576; $\left.P_{\text {adj }}=6.6 \times 10^{-11}\right)$," to name but a few.

In total, there were 1,031 E-aging genes of which half $(\mathrm{n}=502)$ was underrepresented in expression, and the other half (529) was overrepresented. Their GO analysis results are shown in Supplementary File 2. The GO output was small in number and with less
A

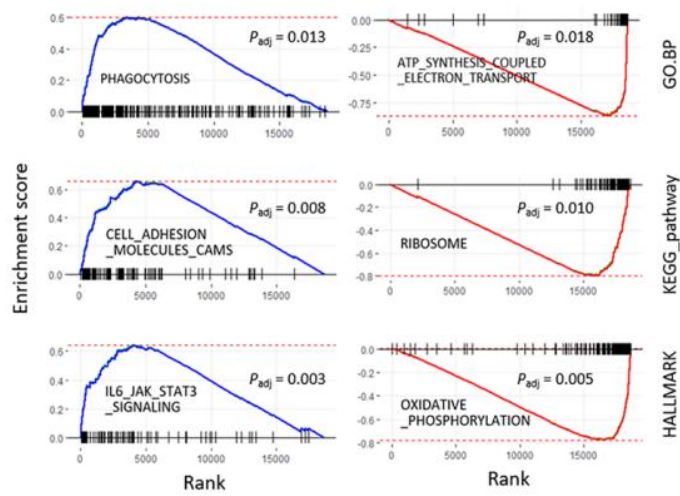

B

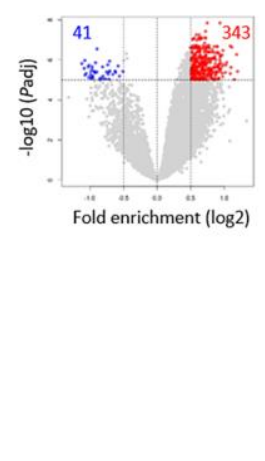

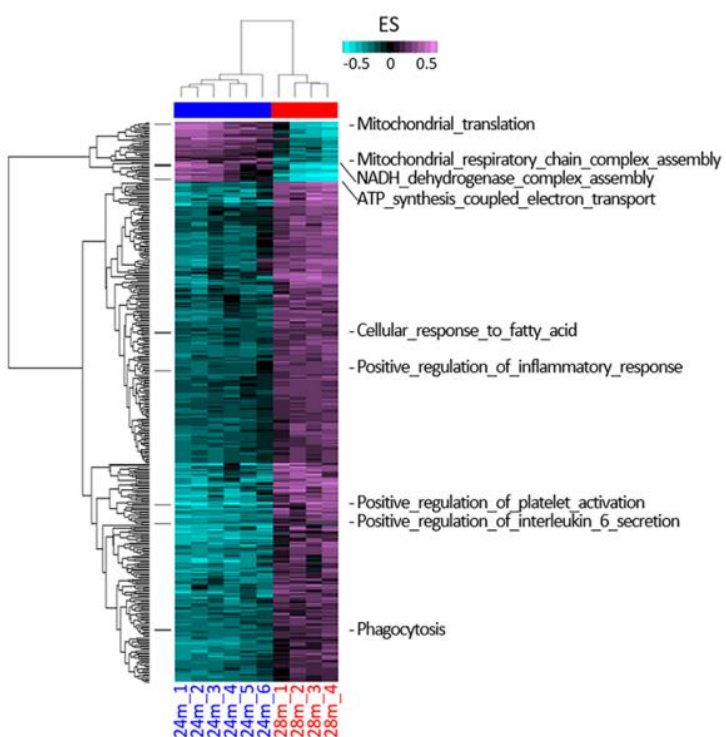

Figure 2. Gene set enrichment analysis (GSEA) using RNA-sequencing data from the skeletal muscle of 24- and 28-month-old mice. (A) The GSEA mountain plots representatively show significant enrichment (left) or depletion (right) of genes, for the indicated gene sets and collections. The thick blue and red lines indicate the running enrichment scores across the fold change-ranked genes (Rank), in comparison to the RNA-seq gene-level expression at 28 over 24 months. Black vertical tick marks below or above the curve indicate the location of individual target genes within the fold change-ranked gene list. Adjusted $P$-values ( $P_{\text {adj, }}$ Benjamin and Hochberg-corrected enrichment statistics) are indicated. (B) Single-sample GSEA with gene sets showing differential enrichment in the skeletal muscle of 24- and 28-month-old mice. Using GSVA, single-sample GSEA was performed on GO.BP collection from MSigDB (v7.0; see Supplementary Figure 1 for other collections). The volcano plot shows the distribution and the number of gene sets with differential enrichment (DE; FDR $<1 \times 10^{-5}$, log2 fold-enrichment $>0.5$ ) between the 24 and 28 months; each dot indicates a gene set in GO.BP MSigDB collection and blue and red dots for depleted and enriched in the 28 months, respectively. The numbers in red and blue indicate the gene set numbers enriched and depleted in $28 \mathrm{~m}$ samples, respectively. Heatmaps show differential enrichments among individual $24 \mathrm{~m}$ and $28 \mathrm{~m}$ samples. Samples were hierarchically clustered on the x-axis (28m, red; $24 \mathrm{~m}$, blue) in an unsupervised manner, and significant DE gene sets are shown on the $y$-axis. Black bars on the left represent the gene sets shown in Figure 4C, and the names of the gene sets are denoted on the right. Colors in the GSVA score bar indicate enrichment scores in individual samples. 
significant terms $\left(\mathrm{P}_{\mathrm{adj}}<0.01\right)$ than the output from the EL-aging genes. For the L-aging genes, only a relatively small number $(n=276)$ were detected. GO analysis of the L-aging genes yielded no significant gene sets, even at $P_{\text {adj }}<0.05$, on the three collections. Therefore, judging from the high statistical significance with which the gene sets were identified, the up- or downturn expression shifts in these EL-aging genes, along with the accompanying up-and-down functions in the corresponding gene sets, might be of no random pattern but have yet to be explored as consequences of the progression of aging. Through hierarchical clustering, we examined changes in the expression levels of EL-aging genes in the identified gene sets. As shown in the heatmaps of the four representative gene sets (Figure 4C), the down- or upturn pattern of age-associated changes was clearly shown by supervised clustering (Figure 5A). In the period spanning the middle age and E-aging (10 to 24 months), the genes either maintained their expression levels relatively constant ("mitochondrial ATP coupled electron transport" and "translation" sets) or showed a gradual decline ("neutrophil-mediated immunity" and "extracellular matrix organization" sets); however, no abrupt change in expression was found among the ELaging genes in this period. Unsupervised clustering data showed that the $28 \mathrm{~m}$ samples were closely associated with the $2 \mathrm{~m}$ samples in all the gene sets (Figure 5B). From the analysis of public mouse muscle transcriptome data [22], we confirmed a similar downturn change in expression levels of "mitochondrial ATP coupled electron transport" gene set with age (Supplementary Figure 4). Since these public transcriptome data came from male mice, we assume no difference in the age-linked expression pattern of ELaging genes between the sexes. To see if there was an age-related change in the mitochondrial copy number in the skeletal muscle, we determined the copy number ratio of the mitochondrial $16 \mathrm{~S}$ gene sequence relative to a nucleus-encoded, single-copy gene sequence (HK2) [23] in the tissue DNAs from 2,10,18, 24, and 28 month old mice that were the same batches of muscle tissues that were used for the transcriptome analysis above. The result indicated that the copy number of mitochondria in
A
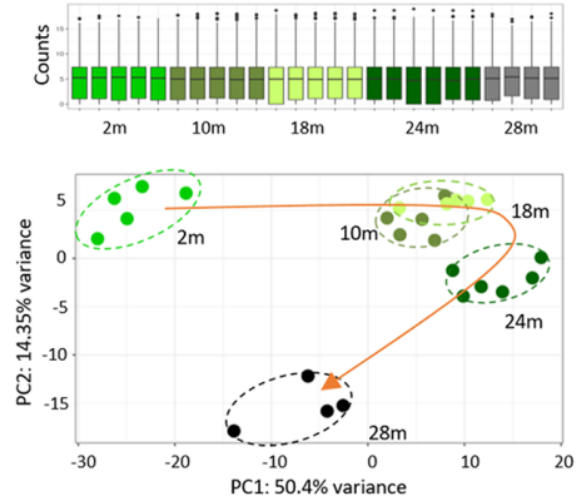

D
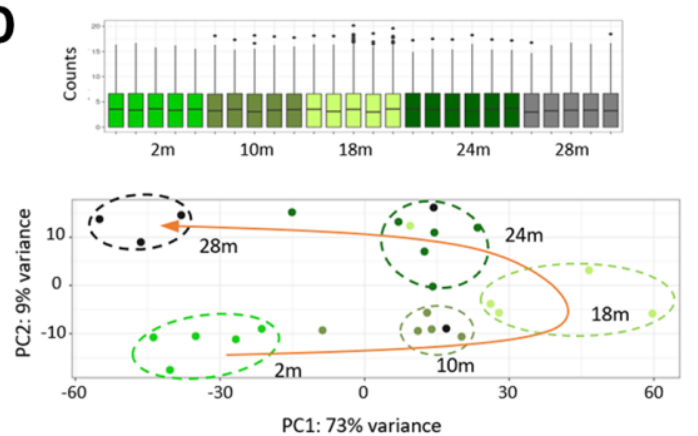

B

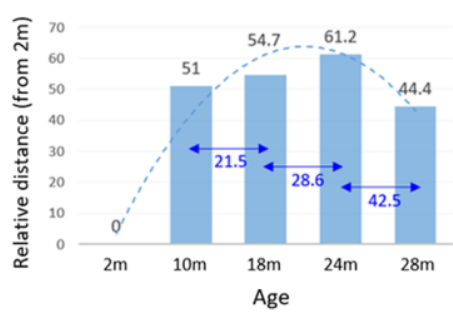

E

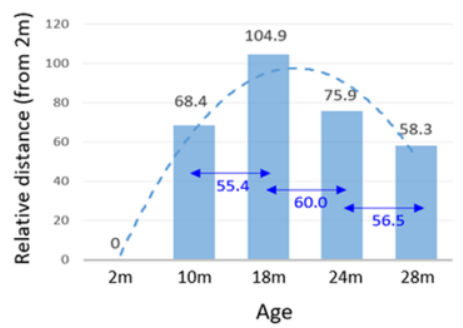

C

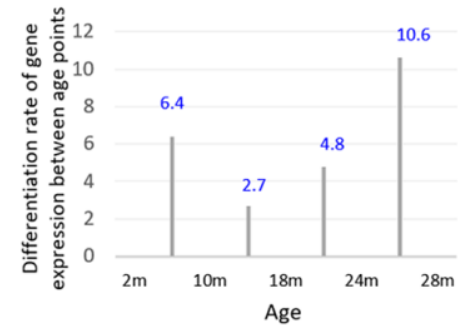

$\mathbf{F}$

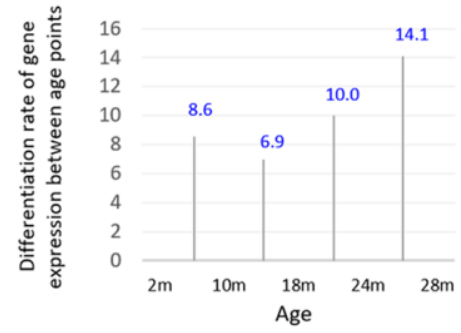

Figure 3. A steep change in the transcriptome of skeletal muscle during the late phase of aging. (A, D) Principal component analysis of the skeletal muscle (A) and peripheral blood mononuclear cell (PBMC); (D) RNA-seq data from 2-, 10-, 18-, 24-, and 28-month-old mice. Transcriptomes of different age groups are marked by different colors. The curved orange arrow connects the group mean transcriptome of each age group to show an age-associated change in the gene expression profile of the skeletal muscle. Box plots show the distribution of normalized counts. (B, E) Measurement of the group mean transcriptomic distance / variance of each age group, relative to the 2-month group (numbers in black) or among the age groups (numbers in blue). (C, F) Per-month differentiation of transcriptomes between the age groups, which divides the transcriptomic distance by the age (month) difference. 
the skeletal muscle showed a downturn pattern of change with age (Supplementary Figure 5), and that the number of mitochondria strongly correlated with the mean expression levels $(r=0.892$; Pearson correlation) of "mitochondrial ATP coupled electron transport" genes. Meanwhile, an assimilation was demonstrated in the PCA plots for the EL-aging genes of the $28 \mathrm{~m}$ group expression profiles, relative to those of the $2 \mathrm{~m}$ group. For the 1,031 E-aging genes, the $28 \mathrm{~m}$ samples overlapped with the $24 \mathrm{~m}$ samples, whereas they overlapped with the $2 \mathrm{~m}$ samples for the 785 downturn and 869 upturn ELaging genes (Figure 5C). This indicated that the transcriptional similarity of old-old muscles with that of young muscles is not restricted to certain gene sets but appears across all the EL-aging genes.

\section{DISCUSSION}

In summary, we observed a comprehensive change in the transcriptome of skeletal muscle during L-aging. The transcriptomes of old-old samples were markedly altered, exhibiting a drastic change in the forward path manifested by the transcriptomes of the younger age groups. Many genes were significantly changed, and of them, the EL-aging genes demonstrated fluctuation in their expression levels with a successive change during the E-aging and L-aging period. However, these changes among old-old samples did not seem to be random but rather synchronic in a variety of gene sets. For example, increasingly expressed EL-aging genes in the L-aging group were significantly enriched in the immunity- and inflammation-related gene sets, whereas decreasingly expressed genes were depleted in the mitochondrial function and translation terms.

We assume that during the E-aging, the EL-aging genes are either in highly expressed or tightly repressed states and multi-layered regulatory systems struggle for transcriptional homeostasis at the expense of cellular energy. During the L-aging, as cellular energy and resources become limited, cells and their transcriptional regulatory systems give way to being decompensated throughout the genome, as evidenced by the upturn and downturn shifts of expression. As such dysregulations over the genome continue unchecked and wide-spread, it eventually results in systemic aging. Likewise, the tension-releasing shift can passively occur in aged, decompensated cells, or there may exist an unknown factor that triggers such changes yet to be identified. At the molecular level, within a cell, genetic and epigenetic regulatory devices that act on the gene sets (Figure 4C) involving EL-aging genes have hitherto managed to homeostatically control the transcriptional milieu over the genes. These devices may break down by increasing stress and tension elicited with aging, leading to the awry expression of genes. Some early EL-aging genes, when altered in expression levels, may accelerate cells to transit to the late phase of aging and further transcriptionally alter the other downstream EL-aging genes. If we could identify these leading EL-aging genes and determine how to keep them safe and unharmed from causes and results of aging, we could
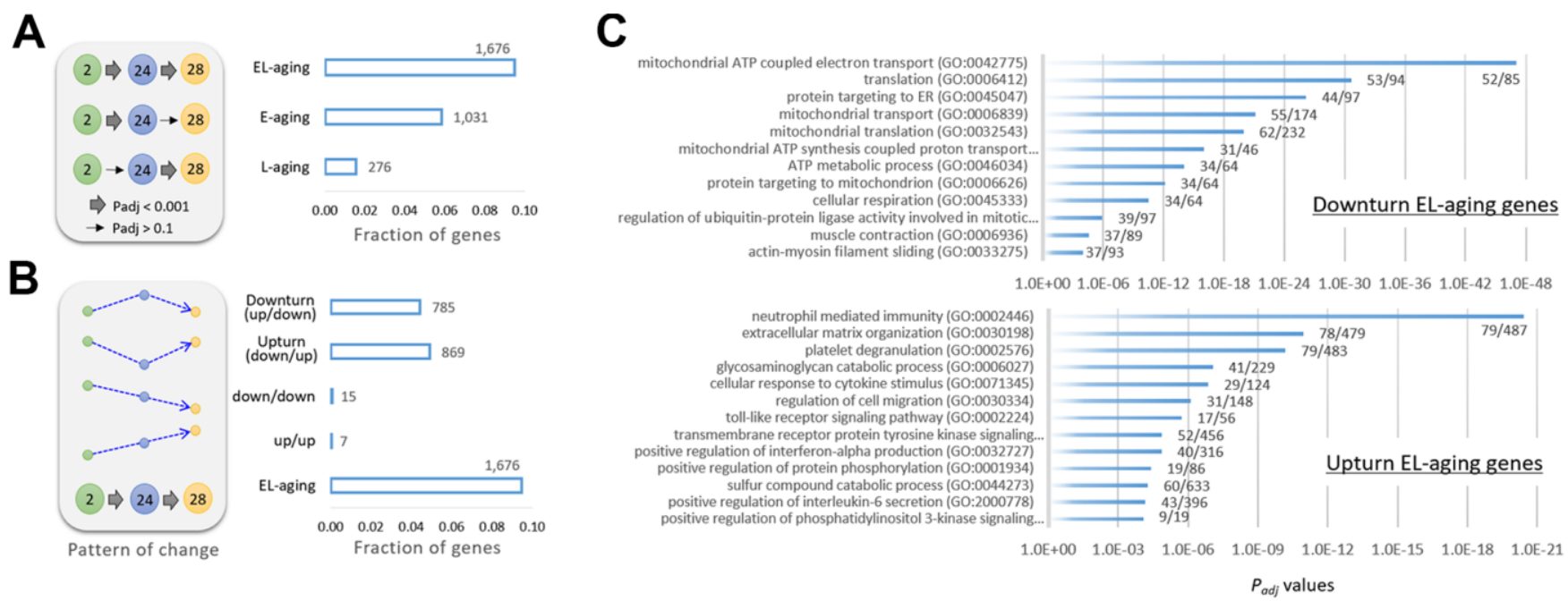

Figure 4. Classification of genes by age-associated expression pattern. (A) Gene categories of E-aging genes, L-aging genes, or ELaging genes that show significant changes in expression levels during E-aging or L-aging, or in both phases (EL-aging), respectively. The thick arrow in the illustration indicates a significant change $\left(P_{\text {adj }}<0.001\right)$ among the age groups, whereas the thin arrow indicates no significance $\left(P_{\text {adj }}>0.1\right)$. (B) Further classification of the EL-aging genes by the pattern (dotted arrows in blue) of change in expression level with age. The number of genes in each category is indicated on the graph. (C) Gene ontology result using 785 downturn (top) and 869 upturn EL-aging genes (bottom). The fractional numbers indicate the number of EL-aging genes over the whole genes in the corresponding gene set. 
delay the oncoming L-aging and prolong the slow Eaging. This can undoubtedly be the genuine way for healthy aging.

There was a substantial change in the gene expression profile of skeletal muscle during L-aging, as visualized by the large swerving trajectory of old-old transcriptomes in the PCA results (Figure 3A). The ELaging genes might play an important part in this process, considering the expression profile of these genes in oldold samples that largely overlapped with those in young samples (Figure 5C). This "reversion-toward-the-young" event is distinguished from the "regression-toward-themean" case often manifested in transcriptomic analysis of aging samples, in that, the latter illustrates antithetical directions of changes occurring between high and low transcribed genes, which results in reducing the gene-to-gene disparities in transcription with age [24]. Regarding an analytical method, whereas the "regression-toward-the-mean" event is obtained from a simple two-point comparison of individual genes with resultant fold-change swaying on the reference time point used, the "reversion-toward-the-young" event could be acquired from a chronological tracing of gene expression levels at multiple time points, thus unwavering and more faithful. In terms of the fraction of (EL-aging) genes showing the "reversion-toward-theyoung" change, 1,676 genes were output after the $P_{\text {adj }}=$ 0.001 cut-off. When the cut-off was lowered to $P_{\text {adj }}=$ 0.05 , the number increased to 3,996 , which is $22.6 \%$ of all the genes, a large fraction enough to call the movement a global pattern. We previously observed this "reversion-toward-the-young" pattern of expression change, among genomic retroelements in mouse skeletal muscle [16]. Most subfamilies of LINE1s and LTRs showed an upturn change in expression levels (apart from the statistical significance of the changes); their initial high-level expression at two months was diminished at 20 months and then increased again at 28 months. Given the scattered presence of retroelement sequences over whole chromosomes and the large fraction of EL-aging genes, we suggest that the agelinked up- and downturn changes in expression are genome-wide trends in aged cells and tissues.

Of course the expression "the reversion-toward-theyoung" does not mean the physical rejuvenation of skeletal muscles; it just portrays the assimilation of $28 \mathrm{~m}$

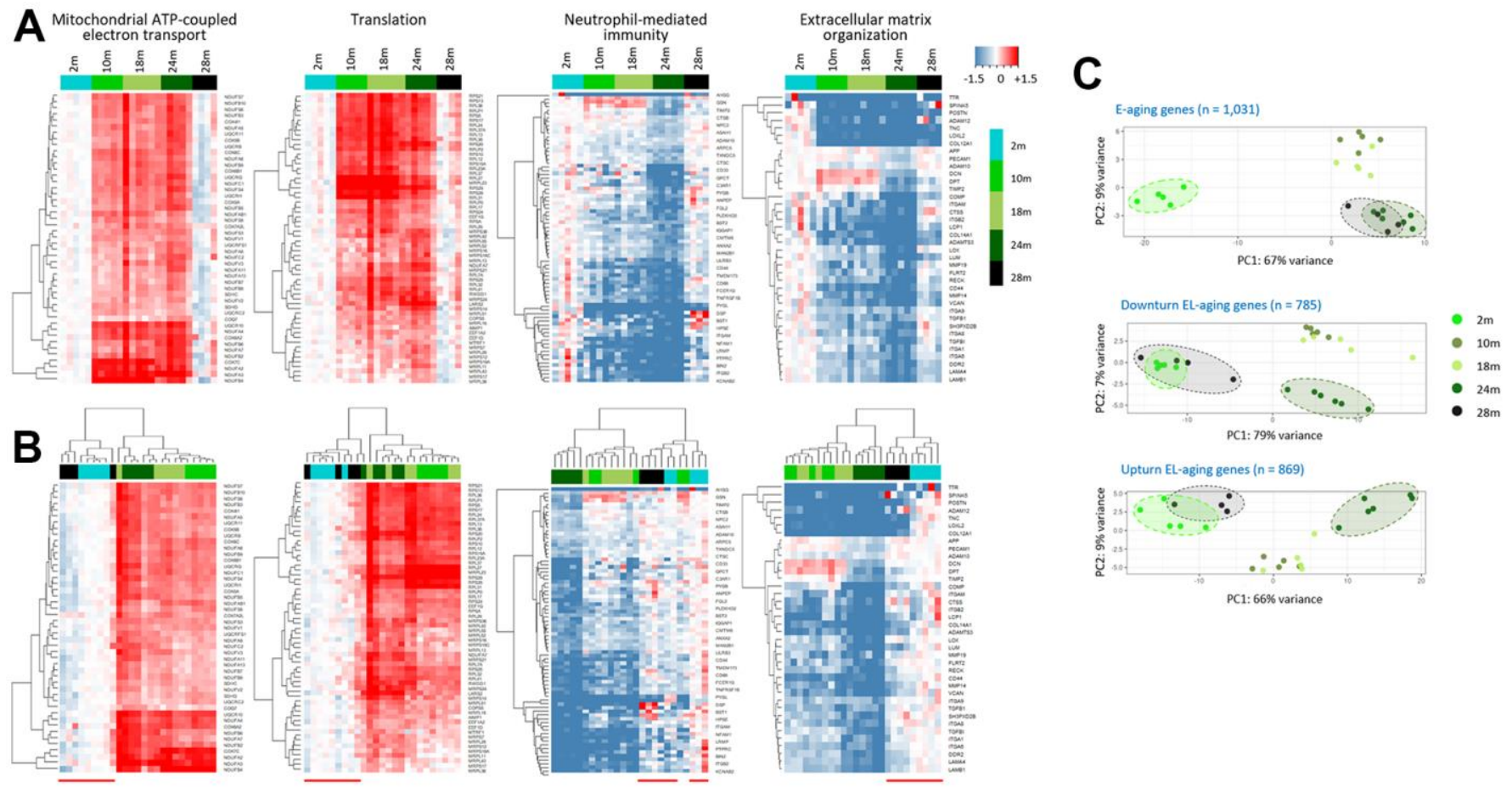

Figure 5. Assimilation of the EL-aging gene expression profiles of the skeletal muscle in super elderly mice with those in the young mice. (A, B) Heatmaps of EL-aging genes for expression levels of individual muscle samples relative to the mean level of the 2-month group. Age-associated expression changes in four representative gene sets are shown by unsupervised (A) or supervised hierarchical clustering (B) Red line below the heatmap in B indicates the cluster of $2 \mathrm{~m}$ and $28 \mathrm{~m}$ samples. (C) Comparison of transcriptomes of E-aging (top) and EL-aging genes (bottom) among the age groups. A close similarity is shown in the $24 \mathrm{~m}$ (dark green) and 28 samples (black) for the E-aging gene group, whereas it was in the $2 \mathrm{~m}$ (light green) and $28 \mathrm{~m}$ samples for the EL-aging gene group and their down- and upturn subsets. 
muscle to $2 \mathrm{~m}$ muscle regarding gene expression profile, which we believe is important in that the expressions of certain sets of genes with specific cellular functions are not individually randomly changed but appear to be coordinately regulated during the late phase of aging. There might be an argument that since mature adult mice are usually defined as 3-6 months old, any differences between 2 months and older time points are confounded by these maturational changes and cannot be ascribed solely to aging. Our result indeed showed a big shift in the expression levels of 'Oxidative phosphorylation' and 'Translation' set genes between $2 \mathrm{~m}$ and $10 \mathrm{~m}$ muscles (Figure $5 \mathrm{~A}$ ), favoring the notion of muscle immaturity in $2 \mathrm{~m}$ samples. It thus recommends to be careful of choosing sample age for comparison of skeletal muscle and to re-consider the use of muscle sample from immature, or mature but still young, mice as a reference. As we made the point clear above, however, we focused on aging events during the late phase of aging and were particularly interested in changes between young-old and old-old. We first found abrupt changes in gene expression levels during the Laging, and next observed the resulting profiles of the changes overall resembling those of $2 \mathrm{~m}$ muscle samples. Hence, our results put no interpretative confusion or ambiguous boundary between aging and maturation changes of sample tissues.

Most age-related diseases are very complex and their etiologies cannot simply be assigned to specific genes; hence, it is hard to match the transcriptional changes of EL-aging genes with certain disorders in the elderly. Nevertheless, the decreasing mitochondrial function and translation (related to the downturn genes) and the increasing inflammation (related to the upturn genes) in the old-old muscle samples accord with the causes of sarcopenia [11]. Sarcopenia is a progressive skeletal muscle disorder involving the accelerated loss of muscle mass and function and occurs commonly as an age-related process in older people, influenced not only by contemporaneous risk factors, but also by genetic and lifestyle factors operating across the life course [25]. In attempt to search for genes associated with sarcopenia and to understand the underlying mechanism driving sarcopenia, several studies investigated changes in muscle gene expression with age. Kang et al. recently identified 15 DEGs from skeletal muscle of sarcopenia patients [26] and, surprisingly, the majority (10/15) of them corresponded with our mouse DEGs (28m vs. 24m; $P_{\text {adj }}<0.05$ ): five DEGs overrepresented (Runxl, Bcl3, Acsl5, Ngfr, and Chi3ll) and five underrepresented (Ndufb5, Tcap, Slc253a, Cycl, and Acat 1 ) in old-old samples. It indicates that the muscle biomarkers identified from human sarcopenia patients are similarly significantly altered in mouse skeletal muscle during L-aging, suggesting that both species share DEGs to a degree in skeletal muscle aging. In addition, from analysis of skeletal muscle transcriptomes, Mahmassani et al. identified 30 DEGs altered by bed rest in old subjects that correlated with change in leg lean mass [27]. Of the 22 DEGs (eight genes were missed in our transcriptome data), 12 genes were also significantly changed in this study during Laging (Arsb, Kctd10, Coq10a, Sort1, Fam96a, Gas1, Thbs4, Avprla, Col22al, Retsat, Ankrd50, and Xpc; $P_{\text {adj }}$ $<0.02$ ). This comparative study supports the notion that those genes we identified as DEGs from the comparison of young-old and old-old samples are implicated in muscle weakening and, possibly, the associated disorders in humans. Meanwhile, sarcopenia is associated with type 2 diabetes in the elderly [28] and, in a re-analysis of public microarray data from human skeletal muscle biopsies, Su et al. found that three agerelated genes were associated with type 2 diabetes [29]; two of them, Cd163 and Gadd45a (the remained Znf415 was missed in our study) were classified as the L-aging $\left(P_{\text {adj }}=1.0 \times 10^{-5}\right)$ and E-aging genes $\left(8.8 \times 10^{-11}\right)$, respectively. In conclusion, what the exact consequences of altered expressions of all these DEGs that were shared in humans and mice are currently unknown. Nonetheless, our transcriptomic signature for the old-old muscle and those failure-prone gene sets acquired by the impact assessment of the late aging phase can be regarded as molecular and physiological traits by which the old-old are distinguishable from the young-old. Therefore, we hope that the 'old-old' muscle transcriptome may serve as a point of reference for some predisposed disorders in the super-elderly.

Previous studies showed different rate of muscle loss between sexes during aging. They recognized malnutrition in females and higher serum myostatin in males as different risk factors for sex-specific difference of muscle aging in humans [30, 31]. We here examined muscle aging in female mice only. So, we cannot exclude the possibility that the males may differ from the females in late phase of aging. Although the analysis of public muscle transcriptome data from male mice suggested no sexual difference in the expression pattern of "mitochondrial ATP coupled electron transport" gene set in life (Supplementary Figure 4), it should be noted that mice (and rat also) have a different survival rate depending on sex and strains (up to four-month difference at $50 \%$ survival age; Strain Survival Information, https://www.nia.nih.gov), which suggests a caution in simple comparison by age between sexes and requires an additional correction for the large gender gap in survival.

In conclusion, we examined the transcriptomes of skeletal muscle obtained from 24- and 28-month-old mice as the young-old and old-old groups, respectively, 
along with 2-, 10-, and 18-month-old mice representing young and midlife controls. Through the subclassification of the old into the young-old and the oldold, we were able to observe a global change in the muscle transcriptome during the late phase of aging, and the changes among the old-old appeared rather synchronic in a variety of functional gene sets. Our results provide a transcriptomic muscle signature that distinguishes old-old mice from young-old mice, which we hope would help to understand skeletal muscle aging in late life and to methodically examine muscle disorders in the elderly with the impact assessment of the late aging phase provided in this study.

\section{MATERIALS AND METHODS}

\section{Ethics statement}

This study was carried out in strict accordance with the recommendations in the Guide for the Care and Use of Laboratory Animals of the National Livestock Research Institute of Korea. The protocol was approved by the Committee on the Ethics of Animal Experiments of the Korea Research Institute of Bioscience and Biotechnology.

\section{Isolation of skeletal muscle and peripheral blood mononuclear cells (PBMCs)}

C57BL/6J female mice at 2, 10, 18, 24, and 28 months of age (four to six in number for each age group) were purchased from LARC (KRIBB) immediately before sacrifice. During organ harvest surgery, neither signs of cachexia nor tumors were found in older mice as well as in younger mice. To obtain skeletal muscle, mice were sacrificed and skeletal muscle in hind limbs were surgically removed and minced. The whole lot of minced tissues were quickly frozen in liquid nitrogen, ground to powder using a mortar and a pestle, and stored in small volumes in $-80^{\circ} \mathrm{C}$ for later use. The powdered tissues were further homogenized using a Biomasher II (DWK Life Sciences) in tissue lysis buffer (ATL buffer; Qiagen) as described before [24]. For collection of PBMCs, the same mice as that used for muscle tissue collection were used. Whole blood was drawn from mouse heart using one $\mathrm{ml}$ syringe and immediately mixed with $2 \mathrm{mg}$ EDTA ( $\mathrm{pH}$ 7.4) per ml of blood to prevent coagulation [32]. The EDTAtreated whole blood was incubated with ten times volume of ACK Lysing Buffer (Thermo) at RT for 10 min to remove red blood cells. The mixture was centrifuged and supernatants were removed. The pellet was resuspended in $5 \mathrm{ml}$ ACK Lysing Buffer to completely remove the residual red blood cells, and PMBCs were collected by additional centrifugation. PBMCs were washed with $1 \mathrm{ml}$ phosphate-buffered saline (PBS) and aliquoted in $1.5 \mathrm{ml}$ tubes to be stored in $-80^{\circ} \mathrm{C}$.

\section{RNA-seq library construction}

Total RNA was extracted from $30 \mathrm{mg}$ of the muscle powder lysed in $200 \mu \mathrm{l}$ of TRIzol Reagent (Thermo). Poly-A tailed RNAs were isolated from $1 \mu \mathrm{g}$ of total RNA using Dynabeads mRNA DIRECT kit (Thermo) according to the manufacture's recommendation. The purified Poly-A tailed mRNAs were treated with DNase I (Sigma) for complete elimination of residual genomic DNAs (gDNA) for $30 \mathrm{~min}$ at $37^{\circ} \mathrm{C}$ prior to RNA-seq library generation. Next, RNA-seq libraries were generated by NEBNext Ultra RNA Library Prep Kit for Illumina (NEB) as described in the provider's protocol. Briefly, the gDNA-free mRNAs were incubated at $94^{\circ} \mathrm{C}$ for $15 \mathrm{~min}$ for fragmentation. First strand cDNA was synthesized with fragmented RNAs using ProtoScript II Reverse Transcriptase and their second strands were synthesized using Second Strand Synthesis Enzyme Mix in the kit before purification. After the end repair of the double-stranded DNAs using NEBNext End Prep Enzyme Mix in the condition of $20^{\circ} \mathrm{C}$ for 30 min and $65^{\circ} \mathrm{C}$ for $30 \mathrm{~min}$, the products were incubated with NEBNext Adaptor and Blunt/TA Ligase Master Mix (NEB) at $20^{\circ} \mathrm{C}$ for $15 \mathrm{~min}$. The resulting ligates were enriched by 12 - 15 cycles of PCR by universal and index primers using $2 \times$ Phusion High-Fidelity PCR Master Mix with HF Buffer (Thermo). Enriched RNAseq libraries were quantified using NEBNext Library Quant Kit for Illumina (NEB), pooled them together by their quantities, and then sequenced by Illumina HiSeqX system ( 2 x 100bp).

\section{RNA-seq data analysis}

Raw sequencing reads were preprocessed to remove adapter sequences and low quality bases using 'Trim_galore v0.6.0' (https://www.bioinformatics.babr aham.ac.uk/projects/trim galore/). Next, the reads were aligned on the reference genome (mm10) using 'STAR v2.7.0' [33], and the resulting SAM files were converted to sorted BAM files using 'samtools v1.9' [34] with '-q 1'. Raw gene expression levels were computed by 'htseq-count v0.11.1' [35], and an expression matrix combined all sample data was generated by a home-brew bash script.

The raw expression dataset was normalized using 'DESeq2 v1.30.0' [36], and gene expression levels between age groups were calculated to identify differentially expressed genes (DEGs, fold-change $>2$, adjusted $P$ value $<0.001$ if not indicated otherwise). For principal component analysis (PCA), the expression dataset was normalized by variance stabilizing 
transformation, and samples were plotted on a 2D PC plain (PC1 x PC2) by 'pcaplot' function (ntop $=5,000$ ) in DESeq2 for visualization. Transcriptomic divergence or distance between age groups, which is based on Euclidean distance, was computed by 'dist' function using whole genes in R. All plots were generated using 'ggplot2' and 'heatmap2' functions in R or MS EXCEL. $\mathrm{R}$ codes for DESeq2 analysis are available in $\langle$ LINK $\rangle$.

\section{Differential expression analysis and GSEA analysis}

For gene set enrichment analysis (GSEA), gene collections for 'HALLMARK', 'KEGG pathways', and 'GO Biological Process' from MSigDB v7.0 [37, 38] were obtained, and gene sets enriched in either 24or 28-months-of-age group were identified using fGSEA [39]. In addition to the fGSEA that was generated based on group means, single-sample GSEA (ssGSEA) was performed using gene set variation analysis (GSVA) [40], a $\mathrm{R}$ package for gene set variation analysis among individual samples. First, the normalized count data from 'DESeq2' were converted to an input matrix for 'GSVA' by a home-brew $\mathrm{R}$ code, and enrichment scores (ES) for individual samples were calculated by the 'gsva' function with 'method=gsva'. Then, using 'limma' [41], a R package for differential expression analysis, gene sets with significantly altered activations (FDR $<1 \times 10^{-5}$ ) were identified, and the results were visualized on volcano plots and heatmaps in $\mathrm{R}$ [42].

\section{RT-PCR and calculation of mitochondrial DNA copy number}

Total RNAs were separately extracted from limb muscle tissues of 24-month- $(\mathrm{n}=6)$ and 28-month-old $(n=4)$ mice as described above and pooled by age group. Reverse transcription was performed by incubating $1 \mu \mathrm{g}$ of DNase I-pretreated RNA with Superscript III enzyme (Invitrogen), $20 \mu \mathrm{M}$ oligo dT primers (Invitrogen), and $50 \mathrm{ng}$ random hexamers (Invitrogen) at $50^{\circ} \mathrm{C}$ for $1 \mathrm{~h}$. Ten ng of the synthesized cDNA was used for a realtime quantitative PCR (QuantStudio3 Real-Time PCR system, $A B I)$ with the specific primers for individual retroelement subfamilies (Supplementary Figure 6). PCR was performed with a following program; 10 minutes of pre-denaturation at $95^{\circ} \mathrm{C}$ followed by 40 cycles of $95^{\circ} \mathrm{C} / 15 \mathrm{sec}$ and $60^{\circ} \mathrm{C} / 1 \mathrm{~min}$. Finally, relative expression level of each gene to Gapdh was calculated using QuantStudio Design and Analysis Software (Thermo).

For estimating the ratio of mitochondrial DNA relative to nuclear DNA, mitochondrial 16S rRNA gene and nuclear-encoded hexokinase 2 (HK2) gene were selected [23]. Using mouse skeletal muscle genomic DNA at different months of age as template and $2 \mathrm{X}$ Power SYBR Green PCR Master Mix (ABI), a quantitative real-time PCR was performed in QuantStudio 3 Real-Time PCR System (ABI). Primers used were 5'-CCGCAAGGGAAAGATGAAAGAC-3' and 5'-TCGTTTGGTTTCGGGGTTTC-3' for $16 \mathrm{~S}$ rRNA gene sequence, and 5'-GCCAGCCTCTC CTGATTTTAGTGT-3' and 5'-GGGAACACAAAA GACCTCTTCTGG-3' for HK2 gene sequence [23]. PCR condition was set as 45 cycles of $95^{\circ} \mathrm{C} / 10 \mathrm{sec}$, $60^{\circ} \mathrm{C} / 10 \mathrm{sec}$, and $72^{\circ} \mathrm{C} / 20 \mathrm{sec}$. The ratio of mitochondrial DNA relative to nuclear DNA was calculated by the classical $\Delta \Delta \mathrm{CT}$ method used for qPCR analysis.

\section{Data availability statement}

The data that support the findings of this study are openly available in Gene Expression Omnibus (GEO) with accession number of GSE.

\section{Abbreviations}

E-/L-aging: early and late phase of aging; $2 \mathrm{~m}$ to $28 \mathrm{~m}$ : two months to 28 months of age; DEG: differentially expressed genes; PCA: principal component analysis; (f)GSEA: (fast pre-ranked) gene set enrichment analysis; ssGSEA: single-sample GSEA; ES: enrichment score; GSVA: gene set variation analysis; PBMC: peripheral blood mononuclear cell; GO: gene ontology.

\section{AUTHOR CONTRIBUTIONS}

YKK designed and supervised the study, performed data mining and analyses, and wrote the manuscript. BM conducted bioinformatic analyses and participated in sample collection. JE participated in sample collection and construction of RNA-seq libraries. JSP participated in mouse husbandry and sample collection. All authors participated in preparation of the manuscript.

\section{CONFLICTS OF INTEREST}

The authors declare that they have no conflicts of interest.

\section{FUNDING}

This work was supported by the grants from the Korea Research Institute of Bioscience Biotechnology (KRIBB) Initiative Program (1711134076) and National Research Foundation of Korea (NRF, Mid-Career Researcher Program). 


\section{REFERENCES}

1. Goldman DP, Cutler D, Rowe JW, Michaud PC, Sullivan J, Peneva D, Olshansky SJ. Substantial health and economic returns from delayed aging may warrant a new focus for medical research. Health Aff (Millwood). 2013; 32:1698-705.

https://doi.org/10.1377/hlthaff.2013.0052

PMID:24101058

2. Alterovitz SS, Mendelsohn GA. Relationship goals of middle-aged, young-old, and old-old Internet daters: an analysis of online personal ads. J Aging Stud. 2013; 27:159-65.

https://doi.org/10.1016/i.jaging.2012.12.006

PMID:23561281

3. Becker G. The oldest old; autonomy in the face of frailty. J Aging Stud. 1994; 8:59-76. https://doi.org/10.1016/0890-4065(94)90019-1 PMID:11774881

4. Halpert BP, Zimmerman MK. The health status of the 'old-old': a reconsideration. Soc Sci Med. 1986; 22:893-9.

https://doi.org/10.1016/0277-9536(86)90162-0 PMID:3738562

5. Mehta M, Whyte E, Lenze E, Hardy S, Roumani $Y$, Subashan P, Huang W, Studenski S. Depressive symptoms in late life: associations with apathy, resilience and disability vary between young-old and old-old. Int J Geriatr Psychiatry. 2008; 23:238-43. https://doi.org/10.1002/gps.1868 PMID:17676651

6. Zanjani FA, Schaie KW, Willis SL. Age group and health status effects on health behavior change. Behav Med. 2006; 32:36-46.

https://doi.org/10.3200/BMED.32.2.36-46

PMID:16903613

7. Divo MJ, Martinez $\mathrm{CH}$, Mannino DM. Ageing and the epidemiology of multimorbidity. Eur Respir J. 2014; 44:1055-68.

https://doi.org/10.1183/09031936.00059814

PMID:25142482

8. Violan C, Foguet-Boreu Q, Flores-Mateo G, Salisbury C, Blom J, Freitag M, Glynn L, Muth C, Valderas JM. Prevalence, determinants and patterns of multimorbidity in primary care: a systematic review of observational studies. PLoS One. 2014; 9:e102149.

https://doi.org/10.1371/journal.pone.0102149 PMID:25048354

9. Barnett K, Mercer SW, Norbury M, Watt G, Wyke S, Guthrie B. Epidemiology of multimorbidity and implications for health care, research, and medical education: a cross-sectional study. Lancet. 2012; 380:37-43.
https://doi.org/10.1016/S0140-6736(12)60240-2 PMID:22579043

10. Harrison DE. Life span as a biomarker. Gerontology: mechanisms of aging. The Jackson Laboratory. https://www.jax.org/research-and-faculty/researchlabs/the-harrison-lab/gerontology/life-span-as-abiomarker 2017.

11. Kim TN, Choi KM. Sarcopenia: definition, epidemiology, and pathophysiology. J Bone Metab. 2013; 20:1-10.

https://doi.org/10.11005/ibm.2013.20.1.1 PMID:24524049

12. Barns M, Gondro C, Tellam RL, Radley-Crabb HG, Grounds MD, Shavlakadze T. Molecular analyses provide insight into mechanisms underlying sarcopenia and myofibre denervation in old skeletal muscles of mice. Int J Biochem Cell Biol. 2014; 53:174-85.

https://doi.org/10.1016/i.biocel.2014.04.025 PMID:24836906

13. Börsch A, Ham DJ, Mittal N, Tintignac LA, Migliavacca E, Feige JN, Rüegg MA, Zavolan M. Molecular and phenotypic analysis of rodent models reveals conserved and species-specific modulators of human sarcopenia. Commun Biol. 2021; 4:194.

https://doi.org/10.1038/s42003-021-01723-z PMID:33580198

14. Erikson GA, Bodian DL, Rueda M, Molparia B, Scott ER, Scott-Van Zeeland AA, Topol SE, Wineinger NE, Niederhuber JE, Topol EJ, Torkamani A. WholeGenome Sequencing of a Healthy Aging Cohort. Cell. 2016; 165:1002-11.

https://doi.org/10.1016/j.cell.2016.03.022 PMID:27114037

15. Flurkey K, Currer JM, Harrison DE. Chapter 20 - Mouse in Aging Research. In The Mouse in Biomedical Research. American College of Laboratory Animal Medicine, The Mouse in Biomedical Research (Second Edition) Academic Press. 2007; 3: 637-72. https://doi.org/10.1016/B978-012369454-6/50074-1

16. Min B, Jeon K, Park JS, Kang YK. Demethylation and derepression of genomic retroelements in the skeletal muscles of aged mice. Aging Cell. 2019; 18:e13042. https://doi.org/10.1111/acel.13042 PMID:31560164

17. Schaum N, Lehallier B, Hahn O, Pálovics R, Hosseinzadeh $S$, Lee SE, Sit R, Lee DP, Losada PM, Zardeneta ME, Fehlmann T, Webber JT, McGeever A, et al, and Tabula Muris Consortium. Ageing hallmarks exhibit organ-specific temporal signatures. Nature. 2020; 583:596-602.

https://doi.org/10.1038/s41586-020-2499-y PMID: $\underline{32669715}$

18. Tabula Muris Consortium. A single-cell transcriptomic atlas characterizes ageing tissues in the mouse. Nature. 
2020; 583:590-5. https://doi.org/10.1038/s41586-020-2496-1 PMID:32669714

19. Barbie DA, Tamayo P, Boehm JS, Kim SY, Moody SE, Dunn IF, Schinzel AC, Sandy P, Meylan E, Scholl C, Fröhling S, Chan EM, Sos ML, et al. Systematic RNA interference reveals that oncogenic KRAS-driven cancers require TBK1. Nature. 2009; 462:108-12. https://doi.org/10.1038/nature08460 PMID:19847166

20. Liu PS. Statistical analysis of next generation sequencing data. (New York: Springer). 2014.

21. Shavlakadze T, Morris M, Fang J, Wang SX, Zhu J, Zhou W, Tse HW, Mondragon-Gonzalez R, Roma G, Glass DJ. Age-Related Gene Expression Signature in Rats Demonstrate Early, Late, and Linear Transcriptional Changes from Multiple Tissues. Cell Rep. 2019; 28:3263-73.e3.

https://doi.org/10.1016/i.celrep.2019.08.043 PMID:31533046

22. Tabula Muris Consortium, Overall coordination, Logistical coordination, Organ collection and processing, Library preparation and sequencing, Computational data analysis, Cell type annotation, Writing group, Supplemental text writing group, and Principal investigators. Single-cell transcriptomics of 20 mouse organs creates a Tabula Muris. Nature. 2018; 562:367-72.

https://doi.org/10.1038/s41586-018-0590-4 PMID:30283141

23. Quiros PM, Goyal A, Jha P, Auwerx J. Analysis of mtDNA/nDNA Ratio in Mice. Curr Protoc Mouse Biol. 2017; 7:47-54. https://doi.org/10.1002/cpmo.21 PMID:28252199

24. Min B, Park M, Jeon K, Park JS, Seo H, Jeong S, Kang YK. Age-associated bimodal transcriptional drift reduces intergenic disparities in transcription. Aging (Albany NY). 2018; 10:789-807.

https://doi.org/10.18632/aging.101428

PMID:29706608

25. Cruz-Jentoft AJ, Sayer AA. Sarcopenia. Lancet. 2019; 393:2636-46.

https://doi.org/10.1016/S0140-6736(19)31138-9 PMID:31171417

26. Kang YJ, Yoo JI, Baek KW. Differential gene expression profile by RNA sequencing study of elderly osteoporotic hip fracture patients with sarcopenia. J Orthop Translat. 2021; 29:10-8.

https://doi.org/10.1016/j.jot.2021.04.009

PMID:34036042

27. Mahmassani ZS, Reidy PT, McKenzie Al, Stubben C, Howard MT, Drummond MJ. Age-dependent skeletal muscle transcriptome response to bed rest-induced atrophy. J Appl Physiol (1985). 2019; 126:894-902. https://doi.org/10.1152/japplphysiol.00811.2018 PMID:30605403

28. Scott D, de Courten B, Ebeling PR. Sarcopenia: a potential cause and consequence of type 2 diabetes in Australia's ageing population? Med J Aust. 2016; 205:329-33.

https://doi.org/10.5694/mja16.00446

PMID:27681976

29. Su J, Ekman C, Oskolkov N, Lahti L, Ström K, Brazma A, Groop L, Rung J, Hansson O. A novel atlas of gene expression in human skeletal muscle reveals molecular changes associated with aging. Skelet Muscle. 2015; 5:35.

https://doi.org/10.1186/s13395-015-0059-1 PMID:26457177

30. Roubenoff $R$, Parise $H$, Payette $H A$, Abad LW, D’Agostino R, Jacques PF, Wilson PW, Dinarello CA, Harris TB. Cytokines, insulin-like growth factor 1 , sarcopenia, and mortality in very old communitydwelling men and women: the Framingham Heart Study. Am J Med. 2003; 115:429-35. https://doi.org/10.1016/i.amjmed.2003.05.001 PMID:14563498

31. Tay L, Ding YY, Leung BP, Ismail NH, Yeo A, Yew S, Tay $\mathrm{KS}$, Tan $\mathrm{CH}$, Chong MS. Sex-specific differences in risk factors for sarcopenia amongst community-dwelling older adults. Age (Dordr). 2015; 37:121.

https://doi.org/10.1007/s11357-015-9860-3 PMID:26607157

32. Park M, Min B, Jeon K, Cho S, Park JS, Kim J, Jeon J, Song J, Kim S, Jeong $S$, Seo $H$, Kang YK. Age-associated chromatin relaxation is enhanced in Huntington's disease mice. Aging (Albany NY). 2017; 9:803-22.

https://doi.org/10.18632/aging.101193 PMID:28288000

33. Dobin A, Davis CA, Schlesinger F, Drenkow J, Zaleski C, Jha S, Batut $P$, Chaisson M, Gingeras TR. STAR: ultrafast universal RNA-seq aligner. Bioinformatics. 2013; 29:15-21.

https://doi.org/10.1093/bioinformatics/bts635 PMID:23104886

34. Li H, Handsaker B, Wysoker A, Fennell T, Ruan J, Homer N, Marth G, Abecasis G, Durbin R, and 1000 Genome Project Data Processing Subgroup. The Sequence Alignment/Map format and SAMtools. Bioinformatics. 2009; 25:2078-9.

https://doi.org/10.1093/bioinformatics/btp352 PMID:19505943

35. Anders S, Pyl PT, Huber W. HTSeq--a Python framework to work with high-throughput sequencing 
data. Bioinformatics. 2015; 31:166-9.

https://doi.org/10.1093/bioinformatics/btu638

PMID:25260700

36. Love MI, Huber W, Anders S. Moderated estimation of fold change and dispersion for RNA-seq data with DESeq2. Genome Biol. 2014; 15:550.

https://doi.org/10.1186/s13059-014-0550-8

PMID:25516281

37. Liberzon A, Subramanian A, Pinchback R, Thorvaldsdóttir H, Tamayo P, Mesirov JP. Molecular signatures database (MSigDB) 3.0. Bioinformatics. 2011; 27:1739-40.

https://doi.org/10.1093/bioinformatics/btr260

PMID:21546393

38. Liberzon A, Birger C, Thorvaldsdóttir H, Ghandi M, Mesirov JP, Tamayo P. The Molecular Signatures Database (MSigDB) hallmark gene set collection. Cell Syst. 2015; 1:417-25.

https://doi.org/10.1016/j.cels.2015.12.004

PMID:26771021

39. Korotkevich G, Sukhov V, Sergushichev A. Fast gene set enrichment analysis. bioRxiv. 2019. [Epub ahead of print]. https://doi.org/10.1101/060012
40. Hänzelmann S, Castelo R, Guinney J. GSVA: gene set variation analysis for microarray and RNA-seq data. BMC Bioinformatics. 2013; 14:7.

https://doi.org/10.1186/1471-2105-14-7

PMID:23323831

41. Ritchie ME, Phipson B, Wu D, Hu Y, Law CW, Shi W, Smyth GK. limma powers differential expression analyses for RNA-sequencing and microarray studies. Nucleic Acids Res. 2015; 43:e47.

https://doi.org/10.1093/nar/gkv007

PMID:25605792

42. Kang YK, Min B. SETDB1 Overexpression Sets an Intertumoral Transcriptomic Divergence in Nonsmall Cell Lung Carcinoma. Front Genet. 2020; 11:573515.

https://doi.org/10.3389/fgene.2020.573515

PMID:33343623 


\section{SUPPLEMENTARY MATERIALS}

\section{Supplementary Figures}
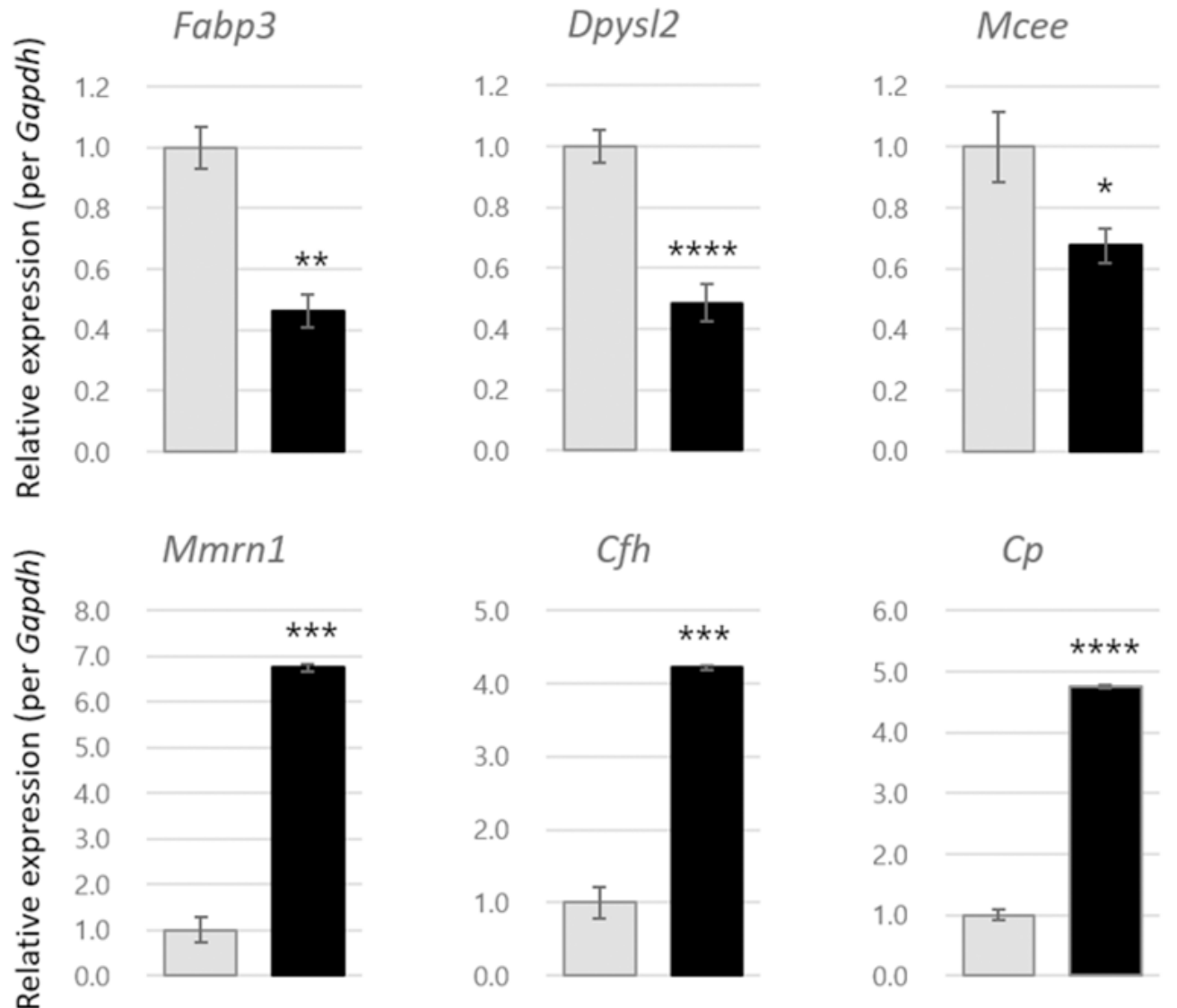

$24 m$
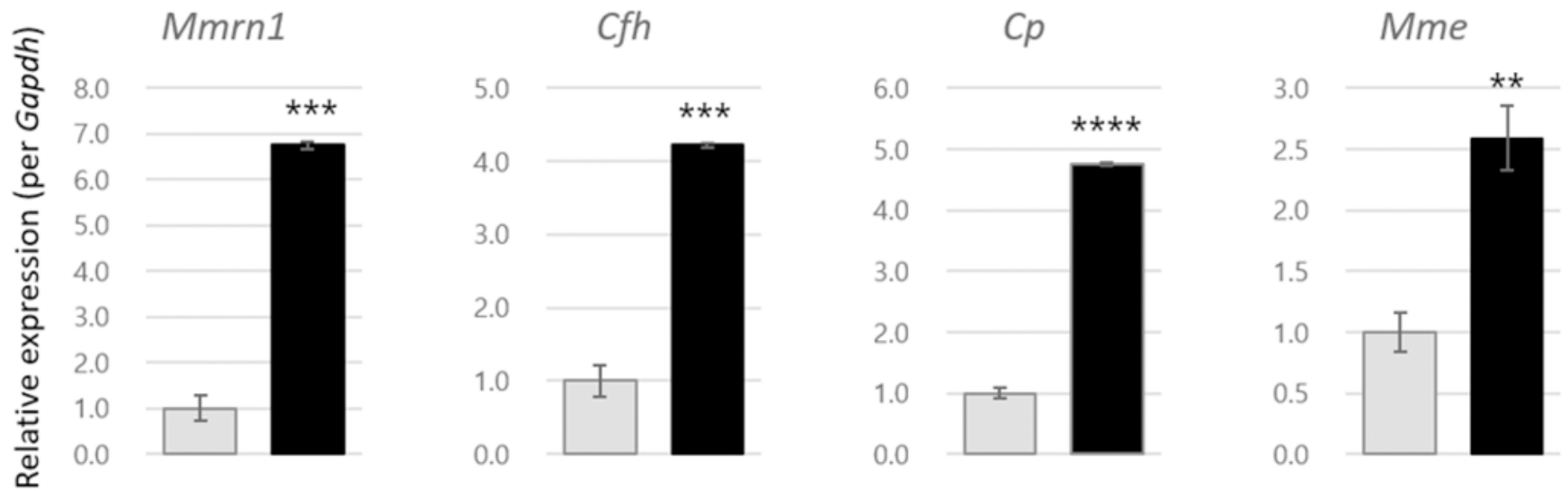

Supplementary Figure 1. Quantitative real-time- PCR for validation of differential expression of genes between the youngold $(24 \mathrm{~m})$ and old-old $(28 \mathrm{~m})$ muscle samples. Asterisks indicate significant different in expression levels. Error bars, standard deviations. Gapdh transcript level was used as an internal control. 
A

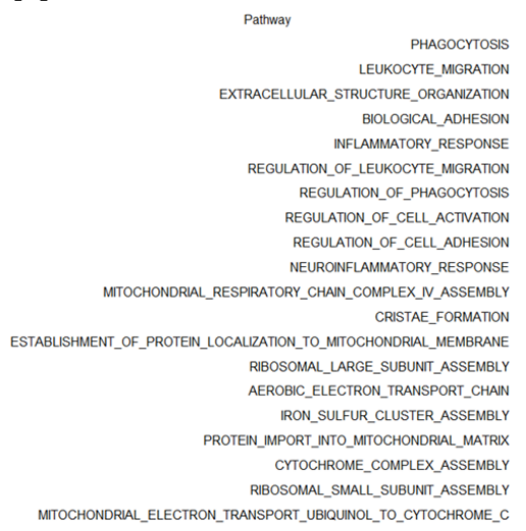

$\underline{\text { GO.BP }}$

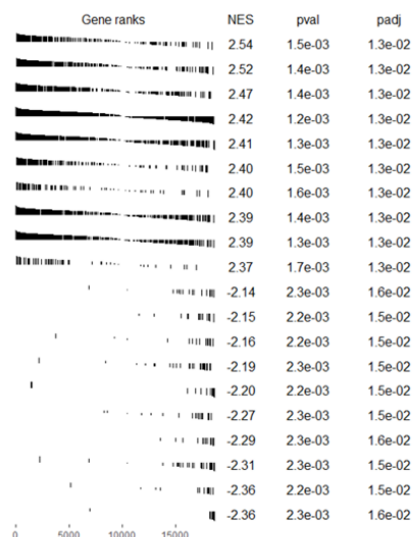

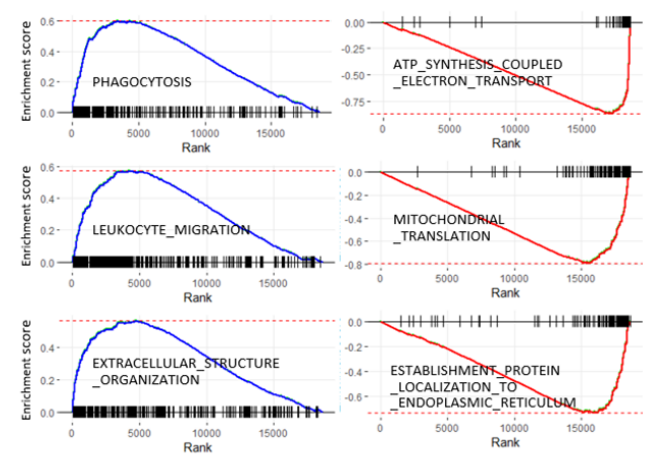

B

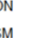

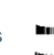
Gene ranks
Nan a...

\section{ON}

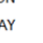

\section{(a) \\ ME}

$$
\text { I' }
$$
SE

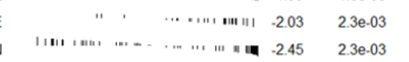

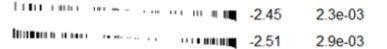

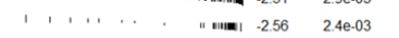

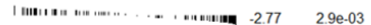

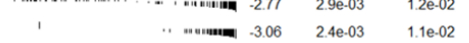

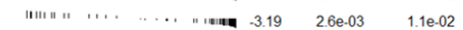

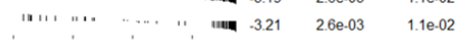

C

Hallmark

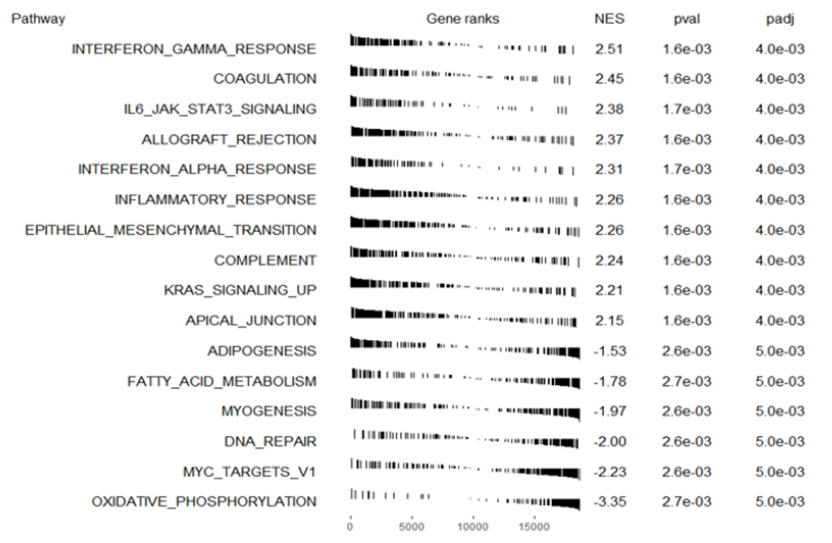

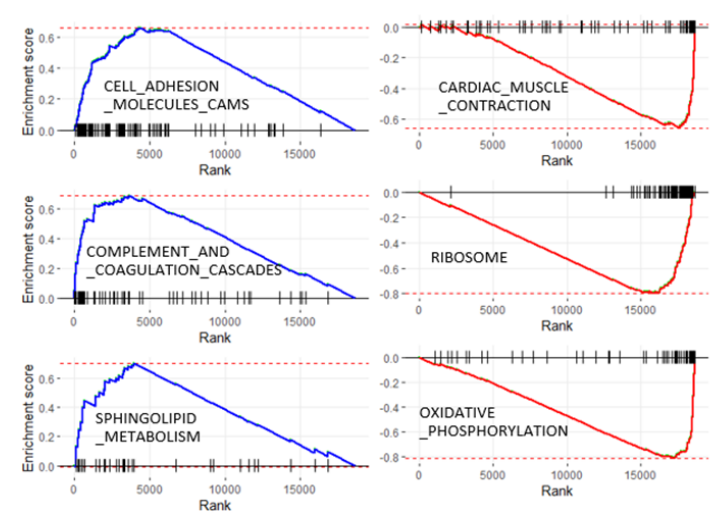

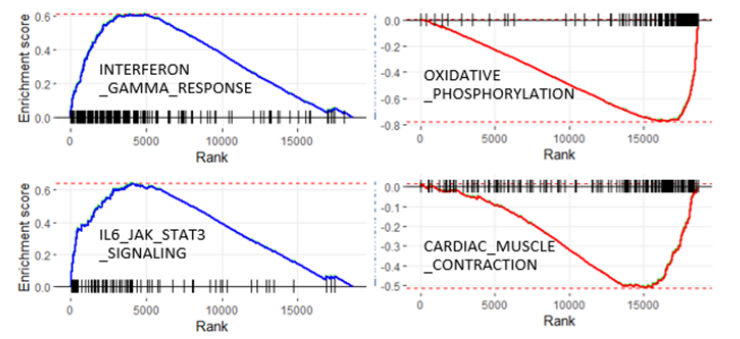

Supplementary Figure 2. Fast pre-ranked gene set enrichment analysis (fG5EA) result on GO. BP (Gene ontology-Biological process; (A), KEGG_pathway (B), and Hallmark (C) collections using RNA-seq data obtained from the mouse skeletal muscle at 24 and 28 months of age. Left panel in (A-C) shows a tabulated result using plotGseaTable function in R, and right panel the GSEA mountain plot which representatively showing a significant enrichment (left) or depletion (right) of genes for indicated gene sets and collections. Thick blue and red lines indicate the running enrichment scores across the fold change-ranked genes (Rank) in comparison between the RNA-seq gene-level expression of 28 months over 24 months. Black vertical tick marks below or above the curve indicate the location of individual target genes within the fold change-ranked gene list. NES, normalized enrichment score. 

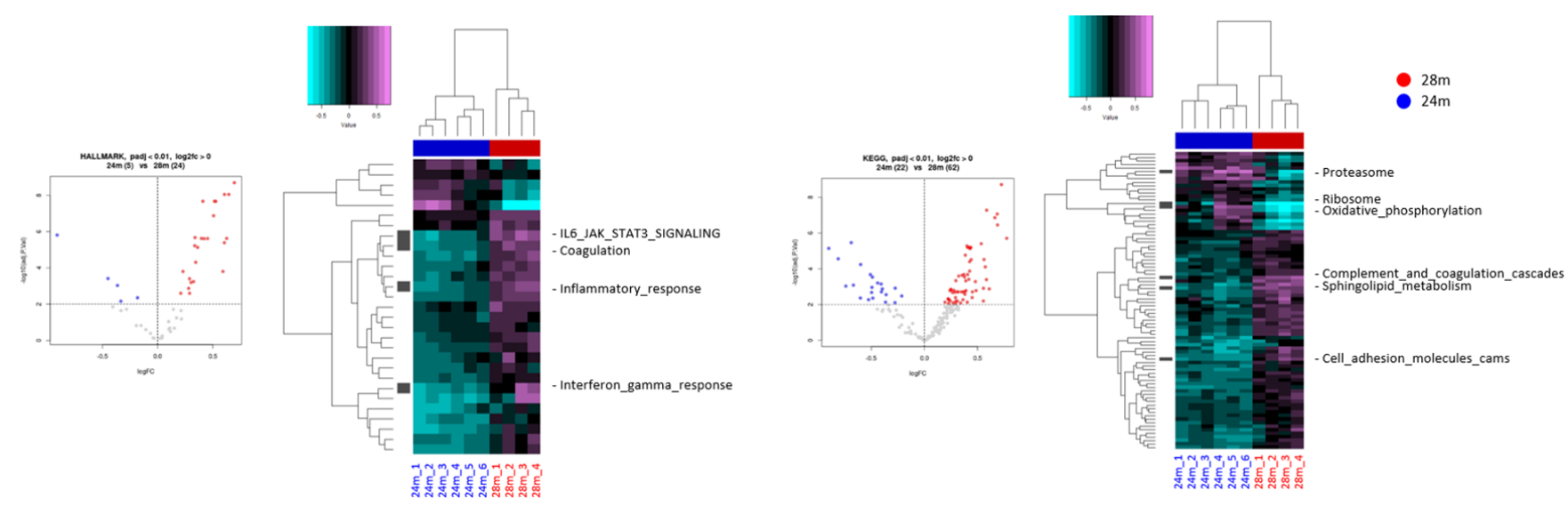

Supplementary Figure 3. Single sample GSEA with gene sets showing differential enrichments in the skeletal muscle of 24- and 28-month-old mice. Using GSVA, single sample GSEA was performed on Hallmark (left) and KEGG_pathway (right) collection. Volcano plot shows the distribution and the number of gene sets with differential enrichments (DE; $F D R<1 \times 10^{-5}$ ) between the 24 months and 28 months; each dot indicates a gene set in each collection, blue and red dots for depleted and enriched in the 28 months, respectively. Heatmaps show the differential enrichments among individual $24 \mathrm{~m}$ and $28 \mathrm{~m}$ samples. Samples are hierarchically clustered on $x$-axis ( $28 \mathrm{~m}$, red; $24 \mathrm{~m}$, blue) in a unsupervised way, and significant DE gene sets are shown on y-axis. Black bars on the left represent the gene sets shown in Figure 4C, and the names of the gene sets are denoted on the right. Colors in GSVA score bar indicate enrichment scores in individual samples.
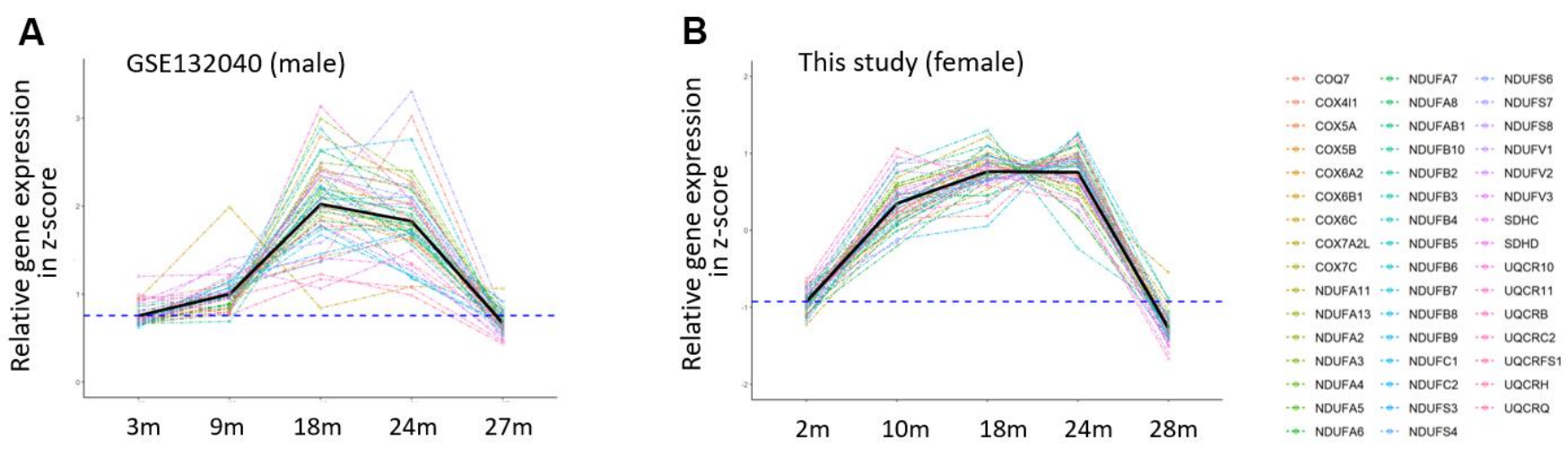

Supplementary Figure 4. Expression patterns of "mitochondrial ATP coupled electron transport" genes with age in skeletal muscle. Gene expression patterns in the public mouse RNA-seq data (GSE132040, A) obtained from male limbs were compared with those in our RNA-seq data (B; female). Black line indicates the mean expression level of the genes and the dotted blue line a reference point (z-score of $3 \mathrm{~m}(\mathbf{A})$ or $2 \mathrm{~m}$ (B) samples). 


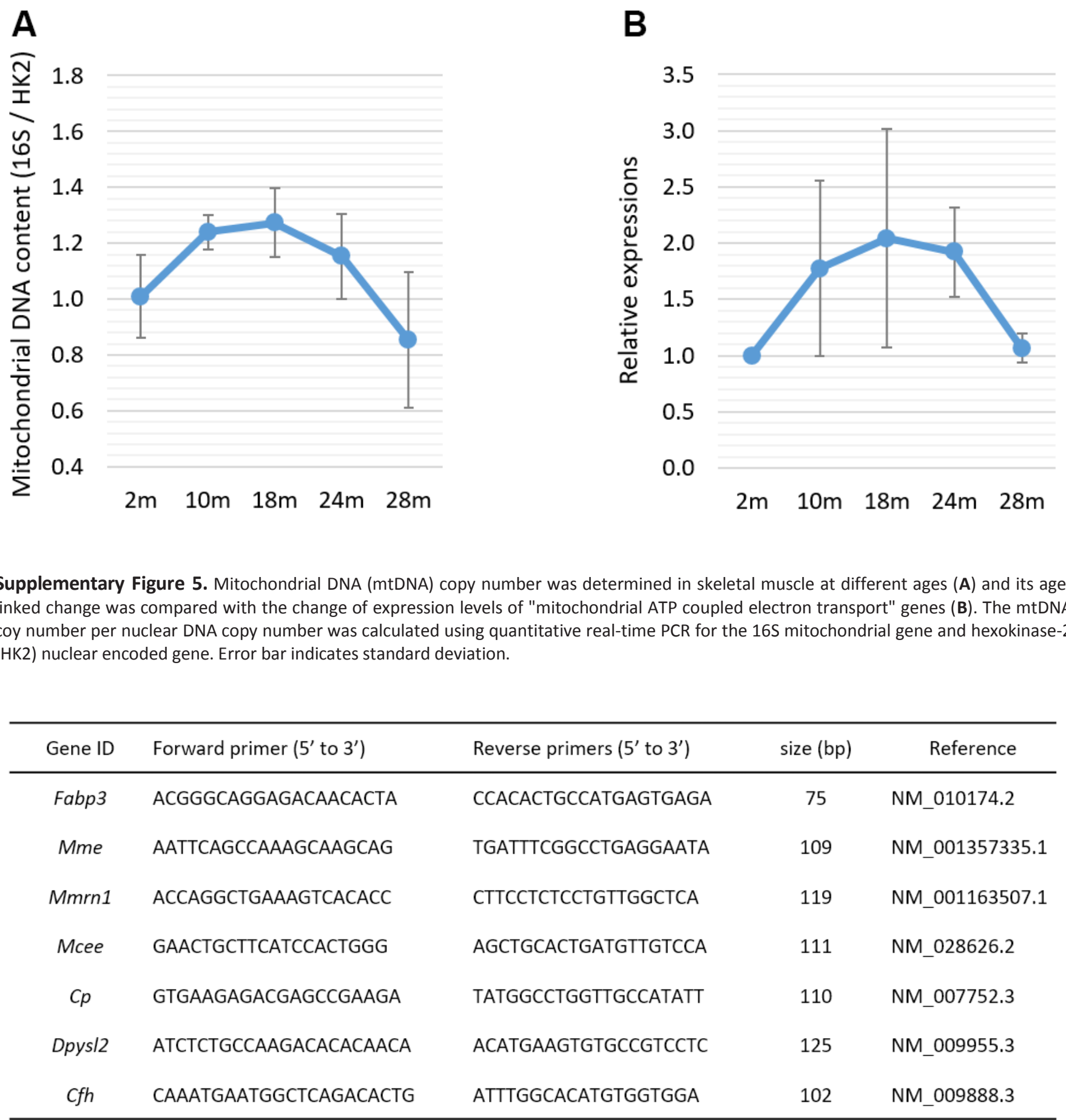

Supplementary Figure 6. Primer sequence information used in quantitative real-time PCR. 


\section{SUPPLEMENTARY FILES}

Please browse Full Text version to see the data of Supplementary Files 1, 2.

Supplementary File 1. Differentially expressed genes among the age groups.

Supplementary File 2. Lists of E-aging, L-aging, and EL-aging genes and their GO results. 